\title{
Machine Learning for Competitive Grain Growth Behavior in Additive Manufacturing Ti6A14V
}

\author{
Jinghao $\mathrm{Li}^{1}$, Manuel Sage ${ }^{1}$, Xianglin Zhou $^{2}$, Mathieu Brochu ${ }^{3}$, Yaoyao Fiona Zhao ${ }^{1 *}$
}

1 Department of Mechanical Engineering, McGill University, Montreal, Quebec, Canada H2A0C3; ${ }^{2}$ State Key Laboratory for Advanced Metals and Materials, University of Science and Technology Beijing, Beijing 100083, China ${ }^{3}$ Department of Mining and Materials Engineering, McGill University, Montreal, Quebec, Canada H3A0C5

\begin{abstract}
Metal additive manufacturing (MAM) technology is now changing the pattern of the high-end manufacturing industry, among which MAM fabricated Ti6Al4V has been far the most extensively investigated material and attracts a lot of research interests. This work established a deep neural network (DNN) to investigate the grain boundary in competitive grain growth for a bi-crystal system, the column $\beta$ grains of Ti6Al4V as an example. Because of the limited number of experimental samples, the DNN is trained based on the data coming from the Geometrical Limited criterion. A series of direct energy deposition experiment using Ti6A14V is carried out under the Taguchi experimental design. The grain boundary angles between the column grains are measured in the experiment and used to evaluate the accuracy of DNN.
\end{abstract}

\section{Introduction}

Titanium alloys are famous for their lightweight, high strength, heat, and corrosion resistant properties. However, titanium is hard to extract as pure metal and normally used for high-value-added products [1]. In the past few decades, titanium and its alloys are found to be suitable for additive manufacturing (AM). To date, there have been only a limited number of commercial alloys used in AM, among which Ti-6Al-4V has been by far the most extensively investigated. This can be attributed to the strong business case of complex and low production volume titanium parts [2]. AM (also known as 3D printing) refers to a process by which digital 3D design is fabricated in a layer by layer fashion. Metal additive manufacturing (MAM) methods based on powder, wire, sliced metal alloys are famous for their flexibility, efficiency, and accuracy [3]. Due to the nature of the AM process which adds material only at the desired place, the lead-time and material waste in AM are reduced to a relatively low level. More importantly, since the tooling is no longer needed, AM unlocks a significant amount of constraints for the designers when designing products with complex geometry [4]. On the other hand, uncertainties in AM from chemical composition, microstructure, texture, and mechanical properties are restricting its development and application [5]. Thus, recognizing the interplay between material deposition phenomena and process factors in AM is crucial for component quality control. Modeling approaches and numerical simulations on multiple length scales and timescales are the ideal tools to save time and experimental cost. These models enable mechanical property predictions from the process and material parameters and serve as a guideline for further component property optimization.

Ti-6Al-4V MAM studies have shown that columnar grain is a common characteristic in the microstructures of fabricated samples [2, 6-13]. These columnar grains are frequently observed to elongate along built direction, approximately parallel to the preferentially growing directions of prior $\beta$ grain (shown in Fig. 1) which is the result of the solidification process that starts at $1660^{\circ} \mathrm{C}$. It involves the first solidification process of the fusion transfer into crystallographic ordered state phase characterized by body-centered cubic (bcc) crystal structure and can be characterized by the reconstruction function of electron back-scattered diffraction (EBSD) technique [14-18]. The substrate or the previous layer of deposited material acts as a preferential nucleation position on which epitaxial growth starts. In normal conditions, an epitaxially orientated type of growth will develop at the partially melted grains from the substrate. The newborn grains will inherit crystallographic effects such as existing grain orientations from existing crystals. This is the so-called 'epitaxial growth' behavior in MAM [19]. Those with the preferentially growing directions align with the thermal gradient direction will stand out from the competitive grain growth and develop even bigger through the layer bonder in MAM process. 

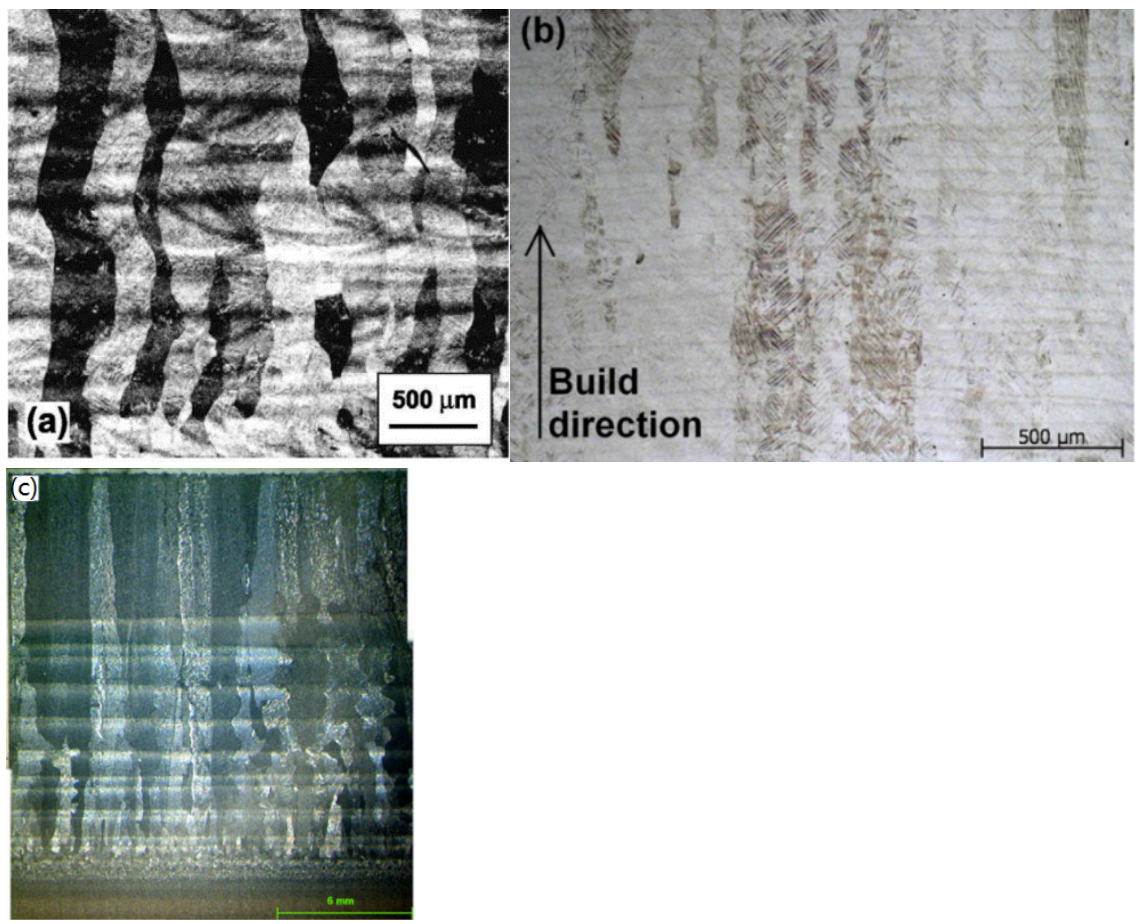

Fig 1: Column grains in Ti-6Al-4V parts produced by (a) DED (b) Selective Laser Melting (SLM) (c) Gas Tungsten Arc Welding (GTAW) [11, 20, 21]

The presence of the column grains has a significant effect on the mechanical properties of the MAM fabricated parts. Literature has shown that cracks have the propensity to propagate along columnar grain boundaries, thus result in anisotropic mechanical properties in as-built condition [22]. Depending on the loading with respect to columnar grain orientation, ductility differences are also discovered in horizontally and vertically built test specimens [14, 16]. However, to represent microstructure including the geometry of column grains, simulation should be carried out involving the grain growth behavior during solidification phenomenon. Solidification is never an easy problem since it involves the phenomenon of the interface transfer and multiple phase transformation. The problem of the competitive grain growth behavior under transient thermal gradient at the melt pool bottom has not been completely solved. This work aims to establish a DNN model to investigate the competitive grain growth behavior in MAM process. DED fabricated Ti-6Al-4V is selected as an example, and the dendritic columnar grains, such as the primary $\beta$ in MAM fabricated Ti-6Al-4V, are referred to as 'grains' or 'column grains' for simplicity.

\section{Model development}

This model is carried out under epitaxial grain growth assumption without nucleation during the material deposition process. In this case, the $\beta$ grains grow preferentially with a solidification direction strongly dependent on the molten pool shape, where the general direction is perpendicular to the solid/liquid interface (the maximum thermal gradient). The melt pool geometry is vital to the resultant microstructure in MAM, and in this work, it is produced by the mathematical modeling which is called "double ellipsoid approximation". The interface of a 3D elliptical sphere melt pool is shown in Fig. 2, the segment of an ellipsoid can be defined in the following equation:

$x^{2} / L^{2}+y^{2} / W^{2}+(z-d)^{2} /(D+d)^{2}=1,(z \leq 0)$

Where $L$ is the length of melt pool tail, with $W$ being half the maximum width, $D$ being the maximum depth of the melt-pool, and dis the displacement in the buildup direction. In the $\mathrm{y}$-z plane ( $\mathrm{x}=0$ ), Eq. (1) becomes

$y^{2} / W^{2}+(z-d)^{2} /(D+d)^{2}=1,(z \leq 0)$

Taking the differential of Eq. (2), we obtain 
$y / W^{2}+((z-d)) /(D+d)^{2} d z / d y=0,(z \leq 0)$

The negative reciprocal value of $\mathrm{dz} / \mathrm{dy}$ is the tangent value of the thermal gradient direction on the $\mathrm{y}$-z plane.

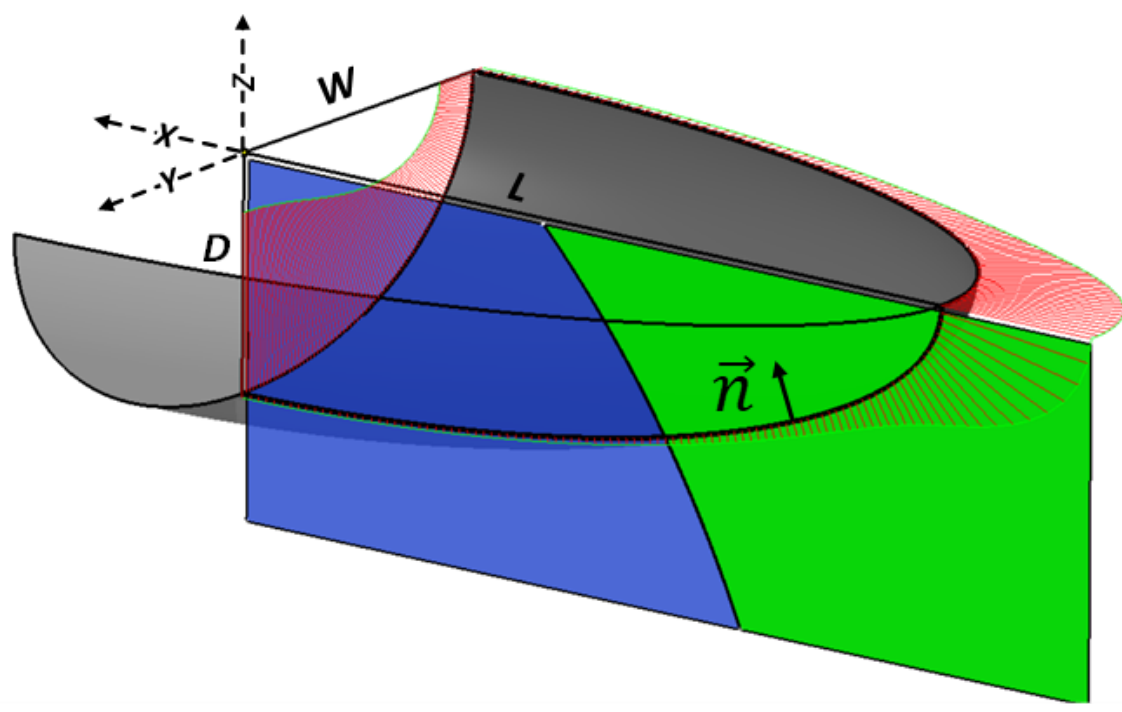

Fig 2: Schematic representation of a $3 D$ melt pool geometry with the normal direction at the melt pool bottom

The principle is the same on the $x-y$ plane and the thermal gradient direction are shown as a red filament in Fig. 2. In the following network experimentation section, the thermal gradient direct is also an input to the network model. The relation between the thermal gradient direction and the crystal orientation are considered, and all the competitive grain growth behavior will be analyzed in a small region between a bi-crystal where thermal gradient direction can be considered as constant.

\section{Network experimentation}

The samples were deposited using a LAWS 1000 automated welding system equipped with a $1 \mathrm{~kW}$ YAG solid fiber laser. The deposition used Ti-6Al-4V ELI wire and was operated under the gas protection environment. Taguchi experimental design is used and the orthogonal arrays are shown in Table 1. The process parameter in terms of laser power, scanning speed and feeding rate is separated into three intensity levels. Further details on the deposition parameters are subject to limited disclosure.

Table 1: Taguchi experimental design 


\begin{tabular}{|c|c|c|c|c|c|c|c|}
\hline \multirow{2}{*}{ Deposition on substrate } & \multicolumn{3}{|c|}{ Deposition on existing layer } \\
\hline \multirow{2}{*}{ Trial } & \multicolumn{3}{|c|}{ Parameters } & \multirow{2}{*}{ Trial } & \multicolumn{3}{|c|}{ Parameters } \\
\cline { 2 - 4 } \cline { 7 - 9 } & A & B & C & & A & B & C \\
\hline 1 & 1 & 1 & 1 & 10 & 1 & 1 & 1 \\
\hline 2 & 1 & 2 & 2 & 11 & 1 & 2 & 2 \\
\hline 3 & 1 & 3 & 3 & 12 & 1 & 3 & 3 \\
\hline 4 & 2 & 1 & 2 & 13 & 2 & 1 & 2 \\
\hline 5 & 2 & 2 & 3 & 14 & 2 & 2 & 3 \\
\hline 6 & 2 & 3 & 1 & 15 & 2 & 3 & 1 \\
\hline 7 & 3 & 1 & 1 & 16 & 3 & 1 & 1 \\
\hline 8 & 3 & 2 & 1 & 17 & 3 & 2 & 1 \\
\hline 9 & 3 & 3 & 2 & 18 & 3 & 3 & 2 \\
\hline
\end{tabular}

Notes: The parameters indicate A: laser power B: scanning speed C: wire feeding rate

To achieve the different cooling condition for the melt pools, the DED experiments are carried out from substrate (specimen 1-9 in Fig.3) and existing layer (specimen 10-18).

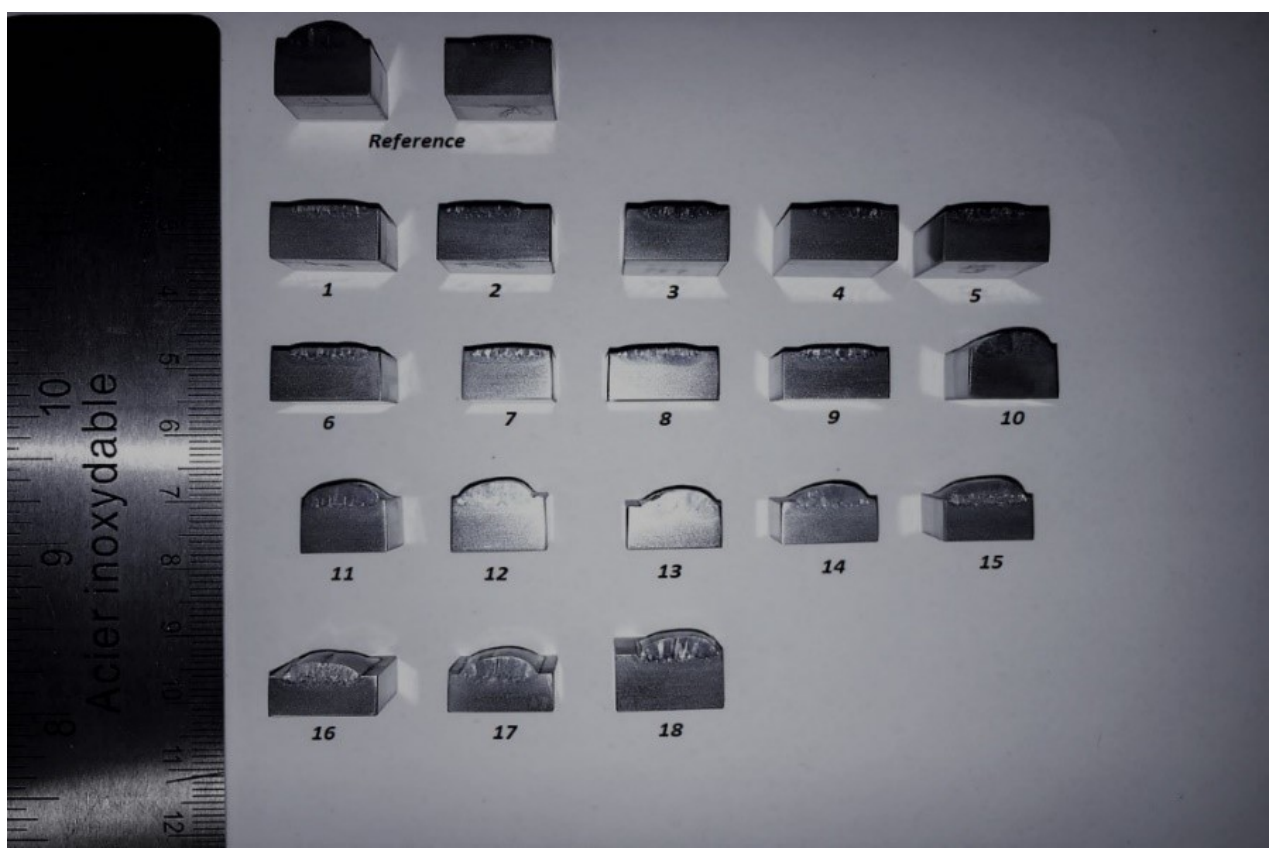

Fig 3: Specimens fabricated via wire DED process under the Taguchi experiment design

For microstructural observation, the as-fabricated samples were ground and then mechanically polished. The polished cross-sections ( $\mathrm{y}-\mathrm{z}$ plane) of the samples were etched in a solution containing $5 \% \mathrm{HF}, 5 \% \mathrm{H}_{2} \mathrm{O}_{2}$ and $90 \% \mathrm{H} 2 \mathrm{O}(\mathrm{v} / \mathrm{v})$ for $10-15 \mathrm{~s}$, and then the metallographic structures were examined using a Keyence digital microscope (optical microscope). 
The columnar $\beta$ grains in DED fabricated Ti6Al4V are generally parallel with the build-up direction, as shown in Fig. 4. The column grains are able to go through the weld bead boundaries (epitaxial grain growth) and achieve a width of $500 \mu \mathrm{m}$ or even several millimeters. The phase distributions were evaluated by electron backscatter diffraction (EBSD) analyses. Under the as-built condition, the solid phase in the column grains is fully martensite with the orthogonal patterns indicating the column grains with different crystal orientations.

Build-up direction

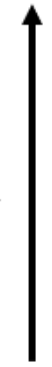

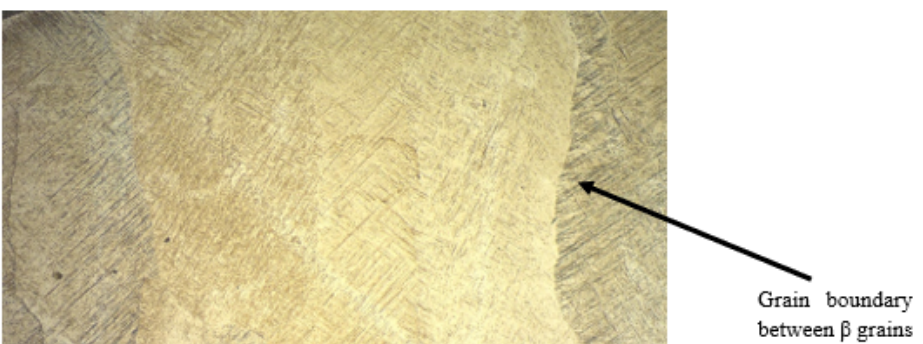

Fig 4: Optical microscope image of columnar $\beta$ grains on the $x-z$ plane

EBSD was conducted using a Hitachi SU-3500 scanning electron microscope equipped with an Oxford EBSD detector, and the results were analyzed using Channel 5. In order to measure the exact crystal orientations of the prior $\beta$ grains, EBSD was carried out near the bottom of the additive portion, shown in Fig. 5. However, the solid phase transformation hides the crystal orientation information of prior $\beta$ grains. So, the reconstruction following the Burgers Orientation Relation (BOR) was carried out using Arpge software [23]. The projection of the preferred grain growth direction (3D vector) on a 2D plane is operated under a self-developed MATLAB code based on the rotation matrix operations of the Euler angles. After this, the crystal orientations which are represented by a 2D vectors (an angle compared to the thermal gradient direction) can be used as inputs to the neural network. Totally 14 groups of data are gathered from the experiment and used for the DNN model evaluation in the next section.

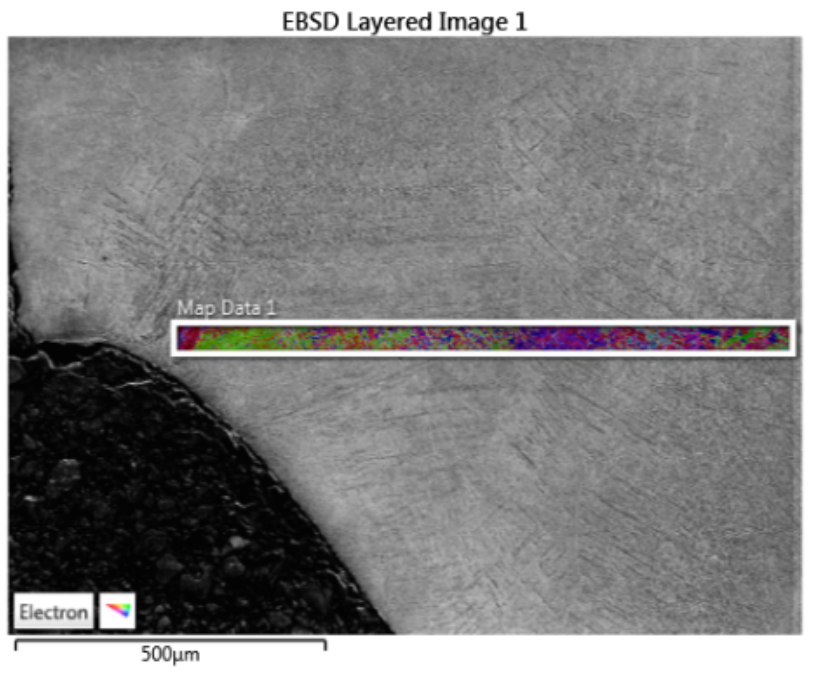

Fig 5: SEM image and EBSD on the additive portion in the $x-z$ plane

4 Feed-forward neural network and results 
The structure of the built feed-forward neural network with two hidden layers is visualized in Fig. 6 [24]. The angle between the thermal gradient direction and the preferred growth direction of a crystal is indicated as $\alpha_{1}$ (left-hand side) and $\alpha_{2}$ (right-hand side).

The DNN model is fed with two input values: $\alpha_{1}$ and $\alpha_{2}$, followed by five and ten neurons in the first and second hidden layer, respectively. The last layer of the fully-connected network consists of a single neuron that outputs the value for the grain-boundary angle. For the implementation of the algorithm, the open source deep learning platform PyTorch was used.

To train the network, around 4,000 instances extracted from Geometrical Limit Criterion (detail can be found in [25]) were taken and divided into training $(80 \%)$ and validation set $(20 \%)$. The mean squared error (MSE) was chosen as a training and evaluation metric. It calculates the squared difference between the target value and the model's output; thus, penalizing far off predictions exponentially stronger.

The goal of the learning process is to determine weight and bias terms for each neuron in the network such that the MSE loss at the output layer is as small as possible. The algorithm achieves that by a technique called backpropagation: Whereas information is fed in forward-mode through the network, the effect of weight and bias terms on the output is computed backward with the help of the derivatives and continuously adjusted during learning. This procedure is displayed for 50 epochs (where one epoch means that all instances in the training set were fed to the network once) of training in Fig. 7.

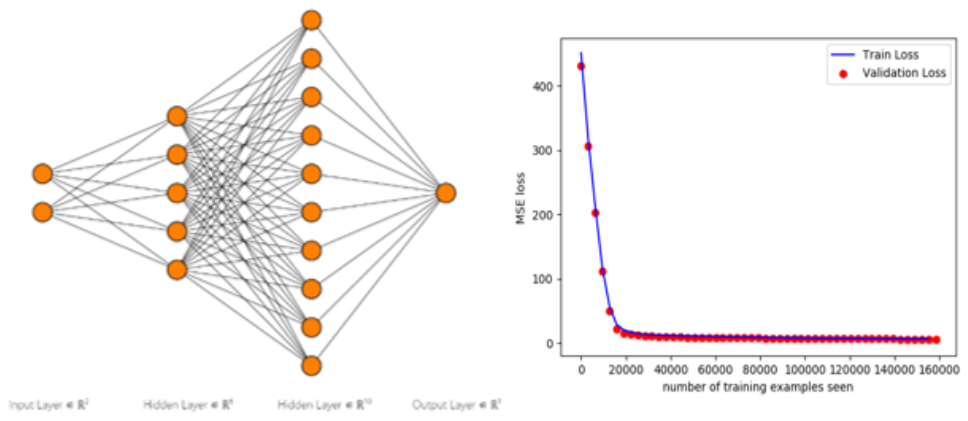

Fig 6: Structure of the neural network used to Fig 7: The evolution of training loss and investigate the grain boundary angles in validation loss during training. competitive grain growth

Besides structure and loss-function, the following settings and hyperparameters were identified to perform the best: Adam, an adaptive optimization algorithm capable of adaptively scaling the learning rate depending on the training progress, was chosen with an initial learning rate of 0.001 [27]. Rectified linear unit function (ReLU) was taken as a non-linear activation function and the model was trained for 1,000 epochs.

The neural network with these settings scored an MSE-loss on training data of 0.23 and 0.30 on validation data. The trained model was then used to predict the grain boundary angle for the 14 instances obtained through experiments and comparing the predicted angles to those obtained from experiments. The corresponding MSE averaged for the 14 instances was 124.17.

The low error values on training and validation data show that the model performs well when predicting the grain boundary angle for formula data. However, an MSE of 124 reveals a significant difference between grain boundary angles predicted with the help of formula data and the experimental results. Taking the square root, this means that among the 14 training samples the prediction is an average of around 11 degrees away from the experimental measurement.

\section{Conclusions}

The DNN model developed in this work from the formula data can be employed to investigate the competitive grain growth behavior in MAM efficiently. A feed-forward neural network is generally an appropriate tool for the task described in this paper. However, the experiments conducted with the neural network showed that a prediction of the grain boundary angle for experimental data is not accurate when formula values are used to train the model. Consequently, this reveals a significant difference between the two data sources: experimental and formula data. This is due to the unique cooling condition in additive manufacturing with a high solidification rate and thermal gradient. 
In future investigations, it is planned to generate more experimental data that will be used not only for evaluation but also for training the neural network. Thereby, the less precise formula data can be replaced gradually, and the accuracy of the model's prediction is expected to improve.

\section{Acknowledgment}

This research was supported by the Natural Sciences and Engineering Research Council of Canada (NSERC) Collaborative Research and Development Grant CRDPJ 479630-15. The lead author also received partial funding from the NSERC Collaborative Research, Training Experience (CREATE) Program Grant 449343, and the National Key Research and Development Program of China (Grant No. 2018YFB0703400). The author would also like to thank McGill Engineering Doctoral Award (MEDA) grant and China Scholarship Council (201706460027).

\section{Reference}

1. http://www.economist.com/node/374784. Dr Chen and the philosopher's stone. 2000.

2. Frazier, W.E., Metal Additive Manufacturing: A Review. Journal of Materials Engineering and Performance, 2014. 23(6): p. 1917-1928.

3. Taufik, M. and P.K. Jain, Role of build orientation in layered manufacturing: a review. International Journal of Manufacturing Technology and Management, 2013. 27(1-3): p. 47-73.

4. Kumke, M., et al., Methods and tools for identifying and leveraging additive manufacturing design potentials. International Journal on Interactive Design and Manufacturing (IJIDeM), 2017.

Greitemeier, D., et al., Uncertainty of Additive Manufactured Ti-6Al-4V: Chemistry, Microstructure and Mechanical Properties. Applied Mechanics and Materials, 2015. 807: p. 169-180.

6. Sames, W.J., et al., The metallurgy and processing science of metal additive manufacturing. International Materials Reviews, 2016. 61(5): p. 315360 .

7. Raghavan, N., et al., Numerical modeling of heat-transfer and the influence of process parameters on tailoring the grain morphology of IN718 in electron beam additive manufacturing. Acta Materialia, 2016. 112: p. 303-314.

Lin, J.J., et al., Microstructural evolution and mechanical properties of Ti-6Al-4V wall deposited by pulsed plasma arc additive manufacturing. Materials \& Design, 2016. 102: p. 30-40.

9. Herzog, D., et al., Additive manufacturing of metals. Acta Materialia, 2016. 117: p. 371-392.

10. Wang, F., et al., Microstructure and Mechanical Properties of Wire and Arc Additive Manufactured Ti-6Al-4V. Metallurgical and Materials Transactions A, 2012. 44(2): p. 968-977.

11. Wang, F., S. Williams, and M. Rush, Morphology investigation on direct current pulsed gas tungsten arc welded additive layer manufactured Ti6Al4V alloy. The International Journal of Advanced Manufacturing Technology, 2011. 57(5-8): p. 597-603.

12. Kobryn, P. and S. Semiatin, The laser additive manufacture of Ti-6Al-4V. Jom, 2001. 53(9): p. 40-42.

13. Thijs, L., et al., A study of the microstructural evolution during selective laser melting of Ti-6Al-4V. Acta Materialia, 2010. 58(9): p. 3303-3312.

14. Obasi, G.C., et al., Effect of $\beta$ grain growth on variant selection and texture memory effect during $\alpha \rightarrow \beta \rightarrow \alpha$ phase transformation in Ti-6 Al-4 V. Acta Materialia, 2012. 60(3): p. 1048-1058.

15. Antonysamy, A.A., P.B. Prangnell, and J. Meyer, Effect of Wall Thickness Transitions on Texture and Grain Structure in Additive Layer Manufacture (ALM) of Ti-6Al-4V. Materials Science Forum, 2012. 706-709: p. 205-210.

16.

Al-Bermani, S.S., et al., The Origin of Microstructural Diversity, Texture, and Mechanical Properties in Electron Beam Melted Ti-6Al-4V. Metallurgical and Materials Transactions A, 2010. 41(13): p. 3422-3434.

17. Thijs, L., et al., Strong morphological and crystallographic texture and resulting yield strength anisotropy in selective laser melted tantalum. Acta Materialia, 2013. 61(12): p. 4657-4668.

18. Antonysamy, A.A., J. Meyer, and P.B. Prangnell, Effect of build geometry on the $\beta$-grain structure and texture in additive manufacture of Ti6Al4V by selective electron beam melting. Materials Characterization, 2013. 84: p. 153-168. 
19. Basak, A. and S. Das, Epitaxy and Microstructure Evolution in Metal Additive Manufacturing. Annual Review of Materials Research, 2016. 46(1): p. 125-149.

20. Kobryn, P.A. and S.L. Semiatin, Microstructure and texture evolution during solidification processing of Ti-6Al-4V. Journal of Materials Processing Technology, 2003. 135(2-3): p. 330-339.

21. Vrancken, B., et al., Heat treatment of Ti6Al4V produced by Selective Laser Melting: Microstructure and mechanical properties. Journal of Alloys and Compounds, 2012. 541: p. 177-185.

22. Kok, Y., et al., Anisotropy and heterogeneity of microstructure and mechanical properties in metal additive manufacturing: A critical review. Materials \& Design, 2018. 139: p. 565-586.

23. Cayron, C., ARPGE: a computer program to automatically reconstruct the parent grains from electron backscatter diffraction data. Journal of applied crystallography, 2007. 40(6): p. 1183-1188.

24. NN-SVG. Available from: http://alexlenail.me/NN-SVG/index.html.

25. Pineau, A., et al., Growth competition between columnar dendritic grains-Cellular automaton versus phase field modeling. Acta Materialia, 2018. 155: p. 286-301.

26. Daalkhaijav, U., et al., Rheological modification of liquid metal for additive manufacturing of stretchable electronics. Advanced Materials Technologies, 2018. 3(4): p. 1700351.

27. Kingma, D.P. and J. Ba, Adam: A method for stochastic optimization. arXiv preprint arXiv:1412.6980, 2014. 